University of Nebraska - Lincoln

DigitalCommons@University of Nebraska - Lincoln

\title{
Characterization of the Aspergillus parasiticus $\Delta 12$-Desaturase Gene: A Role for Lipid Metabolism in the Aspergillus-Seed Interaction
}

\author{
Richard A. Wilson \\ University of Nebraska - Lincoln, rwilson10@unl.edu \\ Ana M. Calvo \\ Northern Illinois University \\ Perng-Kuang Chang \\ USDA-ARS, New Orleans, LA \\ Nancy P. Keller \\ University of Wisconsin - Madison, npk@plantpath.wisc.edu
}

Follow this and additional works at: https://digitalcommons.unl.edu/plantpathfungal

Part of the Plant Pathology Commons

Wilson, Richard A.; Calvo, Ana M.; Chang, Perng-Kuang; and Keller, Nancy P., "Characterization of the Aspergillus parasiticus $\Delta 12$-Desaturase Gene: A Role for Lipid Metabolism in the Aspergillus-Seed Interaction" (2004). Fungal Molecular Plant-Microbe Interactions. 10.

https://digitalcommons.unl.edu/plantpathfungal/10

This Article is brought to you for free and open access by the Plant Pathology Department at DigitalCommons@University of Nebraska - Lincoln. It has been accepted for inclusion in Fungal Molecular PlantMicrobe Interactions by an authorized administrator of DigitalCommons@University of Nebraska - Lincoln. 


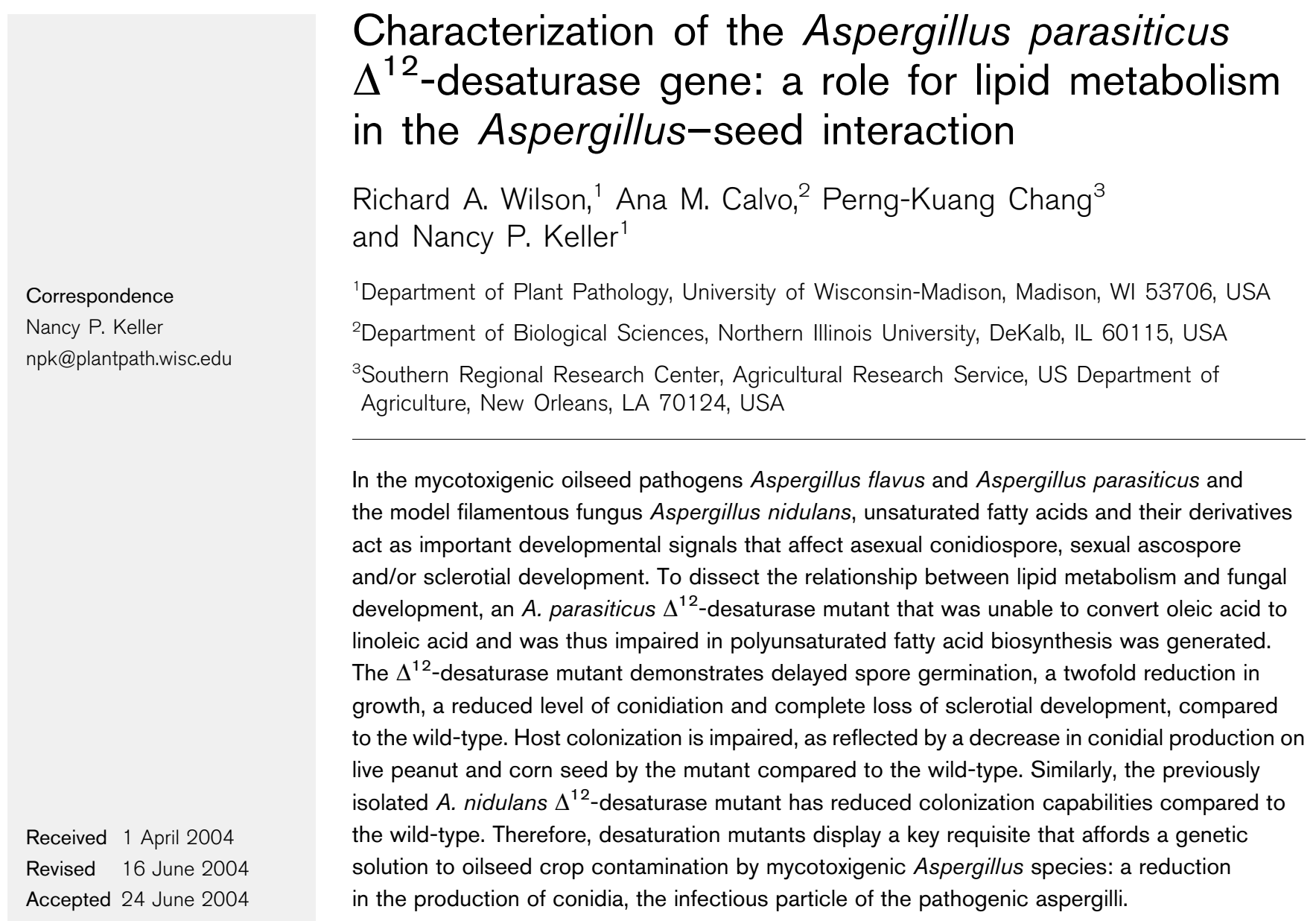

\section{INTRODUCTION}

In agriculture, colonization of oilseed crops by the mycotoxigenic pathogens Aspergillus flavus and Aspergillus parasiticus is a major health and economic concern. Each season, asexual spores called conidia are sources of inoculum for the colonization of crops (Payne, 1992). Two other reproductive structures also play a role in the fungal life cycle. In some species of Aspergillus, sexual ascospores formed in cleistothecia can potentially act as inoculum, whereas mitotic Aspergillus species, such as A. flavus and A. parasiticus, do not produce ascospores and have no known sexual stage, but do produce overwintering structures, sclerotia, which are postulated to be derived from vestigial cleistothecia (Geiser et al., 1996). Recent studies have suggested possible roles for seed- and fungal-derived unsaturated fatty acids in affecting the development of these reproductive structures (Burow et al., 1997; Calvo et al., 1999, 2001). Specifically, linoleic and linolenic acids have been shown to induce asexual

Abbreviations: FAME, fatty acid methyl ester; LOX, lipoxygenase.

The GenBank/EMBL/DDBJ accession number for the sequence reported in this paper is AF528822. sporulation in Aspergillus species (Calvo et al., 1999). Sporulation is also induced by hydroperoxy fatty acid derivatives of linoleic and linolenic acids (Calvo et al., 1999), which are produced by seed lipoxygenases (LOX) during the plant's defensive response to Aspergillus infections (Burow et al., 2000; Wilson et al., 2001). Aspergillus species also produce endogenous hydroxy fatty acids from oleic acid, as well as linoleic and linolenic acids, collectively called psi factor, that regulate spore production (Mazur et al., 1990, 1991; Calvo et al., 2001; Tsitsigiannis et al., 2004; H. W. Gardner \& N. P. Keller, unpublished results).

Oleic and linoleic acids are the most common unsaturated fatty acid components of Aspergillus and oil seeds (Bewley \& Black, 1985; Evans et al., 1986; Calvo et al., 2001). Due to the requirement for oleic acid, linoleic acid and linolenic acid in generating psi factor and LOX products, our investigations have been aimed at understanding the connections between fatty acid metabolism and fungal development and pathogenesis in this host-parasite interaction. In particular, our goals are to generate and characterize Aspergillus mutants that are unable to synthesize polysaturated fatty acids. We previously disrupted the $\Delta^{12}$-oleic acid desaturase gene, odeA (Calvo et al., 2001), in the saprophyte Aspergillus 
nidulans and showed how this mutation leads to almost total loss of polyunsaturated fatty acids and altered conidial and ascospore development. Here, we describe how disrupting an odeA orthologue, ApodeA, in the oilseed pathogen $A$. parasiticus similarly affects fatty acid biosynthesis and results in delayed spore germination, reduced growth and impaired developmental processes in this agronomically important plant pathogen. In addition, this mutant is also severely compromised in its capacity to colonize host corn and peanut seed, as determined by reduced conidiation on seed.

\section{METHODS}

Fungal strains and growth conditions. A. nidulans and A. parasiticus strains used in this study are listed in Table 1. Cultures of both species were grown on A. nidulans glucose minimal medium (GMM) (Calvo et al., 2001) unless otherwise indicated, at $29^{\circ} \mathrm{C}$. Cultures were grown either in the dark or in continuous white light by using an incubator equipped with a General Electric $15 \mathrm{~W}$ broadspectrum fluorescent light bulb (F15T12CW) positioned $50 \mathrm{~cm}$ from the agar surface.

Molecular biology techniques. DNA was extracted from lyophilized mycelium by using standard techniques. For Southern blot analysis, $10 \mu \mathrm{g}$ Pst I restriction-digested genomic DNA was separated by electrophoresis in a $1 \%$ agarose gel and transferred by capillary action to a Hybond membrane (Amersham Biosciences).

Total RNA was extracted from mycelia by using Trizol (Life Technologies). Aliquots of RNA $(10 \mu \mathrm{g})$ were separated on a $1 \cdot 2 \%$ agarose $/ 1 \cdot 5 \%$ formaldehyde gel. RNA was transferred to a Hybond membrane (Amersham Biosciences) by capillary action. fasA gene expression was analysed by using a $0.5 \mathrm{kbp}$ PCR-amplified fragment of $A$. nidulans fasA (GenBank accession no. U75347), which was generated with the primers fasAF $\left(5^{\prime}\right.$-GGATTCCACAGCGG-3') and fasAR (5'-GGGAGCACGGAGAG-3') from genomic DNA of strain RDIT9.32.

DNA fragments to be used as probes were radiolabelled with ${ }^{32} \mathrm{P}$ by using the random primer method (Sambrook et al., 1989). Following prehybridization and addition of the probe, membranes were hybridized overnight at $60^{\circ} \mathrm{C}$ and washed with increasing stringency up to $0 \cdot 1 \times \operatorname{SSC}, 0 \cdot 1 \% \operatorname{SDS}$ at $60^{\circ} \mathrm{C}$.

Identification, sequencing and disruption of the $\Delta^{12}$-oleic acid desaturase-encoding gene, ode $A$, from $A$. parasiticus. The odeA gene was sequenced by using previously described techniques and software (Calvo et al., 2001).

The cosmid pAMC8, containing the A. parasiticus odeA gene, was identified by heterologous hybridization of an A. parasiticus genomic library with a $\Delta^{12}$-desaturase-encoding gene from Candida albicans, as described by Calvo et al. (2001). The sequence of the odeA gene has been deposited in GenBank under accession no. AF528822.

The A. parasiticus odeA disruption vector pAMC37 was created by introducing a pyrG coding-sequence cassette into the ORF of odeA. Firstly, plasmid pBZ5 (kindly provided by Dr J. E. Linz, Department of Food Sciences and Human Nutrition, Michigan State University, East Lansing, MI, USA) was digested to release a $2 \cdot 8 \mathrm{kbp}$ EcoRIHindIII fragment containing the $p y r G$ gene marker from A. parasiticus. The fragment was ligated into pBluescript KS (Stratagene) that had been previously digested with EcoRI and HindIII, generating the plasmid pAMC34. A $4 \mathrm{kbp}$ fragment corresponding to the 5' flanking region of $o d e A$ was amplified from genomic DNA by PCR with proofreading $P f u$ polymerase (Stratagene) and the primers ApodeAF1 ( $5^{\prime}$ GCTGTGAAGCTTCTTCCGCAG-3') and ApodeAR1 (5'-GGTCCGAAGCTTGCTATATCTGG-3'). Thermocycler conditions were $2 \mathrm{~min}$ at $94^{\circ} \mathrm{C}$, followed by 30 cycles of $96^{\circ} \mathrm{C}$ denaturing $(1 \mathrm{~min}), 48^{\circ} \mathrm{C}$ annealing $(1 \mathrm{~min})$ and $72{ }^{\circ} \mathrm{C}$ extension $(4 \mathrm{~min} 30 \mathrm{~s})$. Each of these two primers incorporates a new HindIII site, which were used to ligate the digested PCR product into the HindIII site of pAMC34. Next, a $6 \mathrm{kbp} \mathrm{EcoRI} \mathrm{fragment} \mathrm{corresponding} \mathrm{to} \mathrm{the} 3^{\prime}$ end of the gene was released from pAMC8 and incorporated into the vector at the EcoRI site adjacent to the pyrG cassette, to give pAMC37. Therefore, pAMC37 represents $11 \mathrm{kbp}$ of the gene and flanking regions of odeA, but has $1047 \mathrm{bp}$ of the coding region removed and replaced by $p y r G$. Transformation of the A. parasiticus pyrG $G^{-}$strain CS10 with pAMC37 was performed by using previously described techniques (Skory et al., 1992) and transformants were initially selected based on their ability to grow on GMM lacking the supplements (uracil and uridine) that are required by the $p y r G^{-}$parent.

Physiological studies. Germination studies were performed by inoculating $10^{6}$ spores of each strain into $25 \mathrm{ml}$ liquid GMM and shaking at $29^{\circ} \mathrm{C}$. Colony diameters were measured by pointinoculating $5 \mu \mathrm{l}$ water containing $10^{6}$ spores of either strain onto the centre of plates containing $25 \mathrm{ml}$ GMM agar, followed by growth at $29^{\circ} \mathrm{C}$ for 5 days. Conidial production studies were performed on plates containing $25 \mathrm{ml}$ GMM agar. For each plate, a $5 \mathrm{ml}$ top layer of cool but molten agar that contained $10^{6}$ spores of the appropriate strain was added. For each strain, there was a minimum of four replicate plates. Strains were grown in continuous light or dark for $72 \mathrm{~h}$ (unless otherwise stated) at $29^{\circ} \mathrm{C}$. A core of $15 \mathrm{~mm}$ was removed from the plates at the appropriate time interval and homogenized in $2 \mathrm{ml} 0.01 \%$ Tween 80 in water to release the spores. Spores were counted on a haemocytometer.

To study the effect of the $\Delta o d e A$ mutation on sclerotial production in A. parasiticus, strains were grown on coconut agar medium (CAM) (Trail et al., 1995) and GMM agar. To $30 \mathrm{ml}$ CAM was added a $5 \mathrm{ml}$ top layer of cool but molten agar that contained $10^{6}$ spores of the appropriate strains. For GMM agar, $5 \mu \mathrm{l}$ water containing $10^{6}$ spores of

Table 1. Fungal strains used in this study

\begin{tabular}{|c|c|c|c|}
\hline Strain & Species & Genotype & Source \\
\hline RRAW5.2 & A. nidulans & $\arg B 2 ; \triangle \operatorname{ode} A:: \arg B ; v e A$ & Wilson et al. (2004) \\
\hline RDIT9.32 & A. nidulans & Prototroph & $\begin{array}{l}\text { D. Tsitsigiannis, Department of Plant Pathology, } \\
\text { University of Wisconsin, USA }\end{array}$ \\
\hline ATCC 36537 & A. parasiticus & $w A ;$ ver 1 & American Type Culture Collection \\
\hline CS10 & A. parasiticus & $w A ; \operatorname{ver} 1 ; \operatorname{pyr} G^{-}$ & Skory et al. (1992) \\
\hline TAMC37.41 & A. parasiticus & $w A ; \operatorname{ver} 1 ; \operatorname{pry} G^{-} ; \Delta o d e A:: \operatorname{pyr} G$ & This study \\
\hline
\end{tabular}


either strain was point-inoculated onto the centre of each plate. Plates were incubated in the dark at $29^{\circ} \mathrm{C}$ for 14 days for CAM and 7 days for GMM agar. After this time, plates were sprayed with ethanol to flatten conidia and total numbers of sclerotia per plate were counted by using a dissecting scope. Both CAM and GMM agar experiments were carried out with four replicates.

Colonization studies. Seeds of the near-isogenic Sunrunner and SunOleic97R peanut lines were kindly provided by Dr C. Holbrook (USDA, GA, USA). Sunrunner has $50 \%$ oleic acid and $30 \%$ linoleic acid content, whilst SunOleic97R is a high oleic acid line that has $80 \%$ oleic acid and approximately $2 \%$ linoleic acid content. Prior to infection by $A$. parasiticus or A. nidulans strains, the seeds were shelled, the cotyledons separated and the embryo removed. Seeds from both peanut lines were weighed so that all the peanuts used were between 0.4 and $0.6 \mathrm{~g}$ in weight. For dead-seed experiments, the cotyledons were autoclaved in a liquid cycle for $30 \mathrm{~min}$. For living-seed experiments, cotyledons were surface-sterilized by immersion in $10 \%$ Clorox bleach for $1 \mathrm{~min}$, followed by immersion in sterile distilled water for $1 \mathrm{~min}$. For A. parasiticus, the seeds were inoculated as follows: for each strain, four peanut cotyledons were placed in a $50 \mathrm{ml}$ Falcon tube and to this was added $500 \mu \mathrm{l}$ sterile distilled water containing $10^{6}$ spores of the appropriate strain. Each tube was vortexed for $1 \mathrm{~min}$, the caps were loosened and the samples were placed in the dark at $29^{\circ} \mathrm{C}$ for 4 days. These experiments were performed with four replicates. Simultaneously, GMM agar plates were inoculated, using the top-layer method described above, with the same spore suspensions that were used to infect the peanut seed (at a rate of $10^{6}$ spores per plate, four replicates for each) and placed in the incubator beside the infected seed. After 4 days, spores on the plates were counted as described above. Spores from the infected seed were released by adding $5 \mathrm{ml}$ Tween 80/water to each Falcon tube, vortexing for $1 \mathrm{~min}$ and using an aliquot of this for counting using a haemocytometer.

A. nidulans does not readily infect peanut seed and so the inoculation protocol was modified as follows. One cotyledon was immersed in $1 \mathrm{ml}$ sterile distilled water containing $10^{6}$ spores of the appropriate strain for $30 \mathrm{~min}$, with vigorous vortexing every $10 \mathrm{~min}$. This was repeated six times for each experiment. After $30 \mathrm{~min}$, the infected peanut seeds were placed in a glass Petri dish containing water-saturated filter paper and a water reservoir and left in the dark at $29^{\circ} \mathrm{C}$ for 4 days (in the case of dead seed) and 6 days (in the case of live seed). Plates of GMM agar that were inoculated with the same strains were placed in the incubator simultaneously and counted at the same time points as the infected seed. Spores were released from the seed by using Tween 80/water and vortexing for $1 \mathrm{~min}$. Four cotyledons were used for counting per treatment.

Corn seeds were surface-sterilized as described above. Four seeds were placed in one $50 \mathrm{ml}$ Falcon tube and inoculated as described above for A. parasiticus. Tubes were placed at $29^{\circ} \mathrm{C}$ under dark conditions for 4 days. Each experiment was performed with four replicates.

In all seed-inoculation experiments, Koch's postulates were determined after spore-counting to verify the identity of the Aspergillus strains infecting the seed.

Fatty acid analysis. Fatty acid methyl esters were generated from Aspergillus mycelium for GC analysis as follows. After growth in GMM for $72 \mathrm{~h}$ under conditions of constant light, mycelia were lyophilized and ground into fine powder and the lipids were extracted three times in chloroform: methanol $(2: 1)$ following the method of Bligh \& Dyer (1959). Heptadecanoic acid (17:0; 0.64 ng) was added as an internal standard. The samples were dried down, resuspended in $1 \mathrm{ml} 5 \% \mathrm{HCl}$ in $90 \%$ methanol and placed at $95^{\circ} \mathrm{C}$ for $30 \mathrm{~min}$ to generate fatty acid methyl esters (FAMEs). The methylating reaction was quenched with water and the FAMEs were extracted three times in hexane. The samples were concentrated and a $1 \mu \mathrm{l}$ aliquot of the hexane layer was examined by GC. Identification of peaks was achieved by comparison of sample retention times to those of palmitic, palmitoleic, stearic, oleic, linoleic and linolenic acid standards (FAME-GC mix; Sigma).

Statistical analysis. Spore data and colony diameters were evaluated by analysis of variance (ANOVA) using the Statistical Analysis system (SAS Institute, Cary, NC, USA).

\section{RESULTS}

\section{Isolation and characterization of the $A$. parasiticus odeA gene}

Cosmid pAMC8, which hybridized to the C. albicans odeA gene, was identified from the $A$. parasiticus genomic library. Sequencing of hybridizing regions of pAMC8 and BLAST analysis of these regions revealed the presence of a putative $\Delta^{12}$-desaturase gene (GenBank accession no. AF528822). Further comparison of the A. parasiticus genomic DNA sequence to the cDNA sequence obtained by RT-PCR (accession no. AY267349) showed that the A. parasiticus odeA gene contained one intron and encoded a predicted protein of $466 \mathrm{aa}$. The OdeA protein contained the conserved histidine clusters that are common to $\Delta^{12}$ desaturases and demonstrated 99 and $86 \%$ sequence similarity to the A. flavus (AY280867) and A. nidulans (AF528822) OdeA proteins, respectively, at the amino acid level.

\section{Disruption of the A. parasiticus odeA gene}

The $\Delta^{12}$-oleic acid desaturase gene of $A$. parasiticus was disrupted by homologous recombination as depicted in Fig. 1a. A rapid screen of $96 \mathrm{pyr} \mathrm{G}^{+}$transformants for integration of $p y r G$ at the odeA locus was employed. Undigested genomic DNA from these transformants was run out on an agarose gel and probed with the $0.7 \mathrm{kbp}$ BamHI-EcoRI internal fragment of odeA, shown hatched in Fig. 1a. One transformant, TAMC37.41, did not hybridize to the odeA probe (Fig. 1b, lane 2), indicating removal of the odeA coding region from the genome. Single-copy integration of $p y r G$ at the odeA locus in TAMC37.41 was subsequently confirmed by Southern blot analysis following PstI restriction digestion, using the full-length odeA coding region as a probe, in addition to DNA sequencing and PCR analysis (data not shown).

\section{Fatty acid profile of the $A$. parasiticus $\Delta$ ode $A$ mutant}

To examine the effect of the $\Delta o d e A$ mutation on fatty acid biosynthesis, mycelial fatty acid content of the $A$. parasiticus $\Delta o d e A$ strain was compared to that of the wild-type. Table 2 shows the total amount of fatty acids produced by the mutant and wild-type, as well as the proportion of each fatty acid detected in the FAME mixture. Two major observations were evident from this table. Firstly, the $\Delta o d e A$ mutant accumulated approximately four times more 
(a)

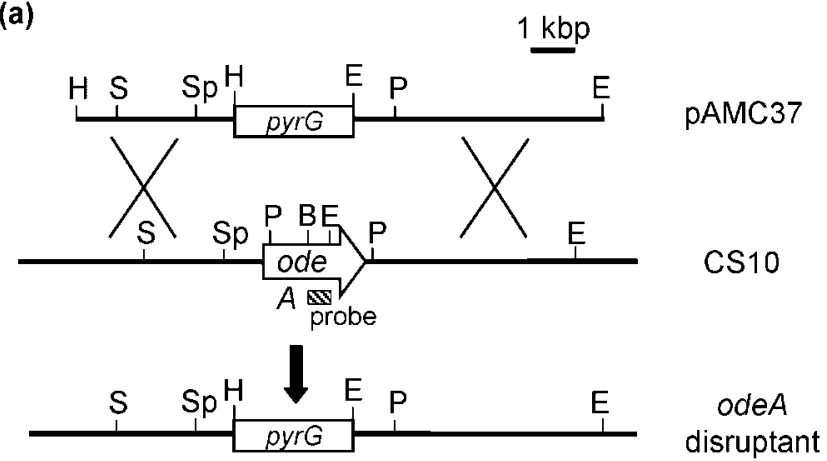

(b)

$1234 \mathrm{kbp}$

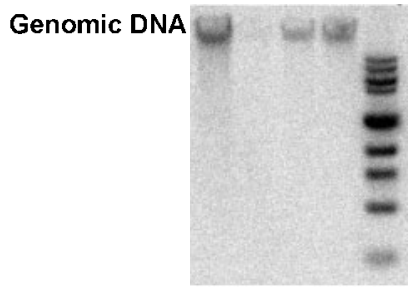

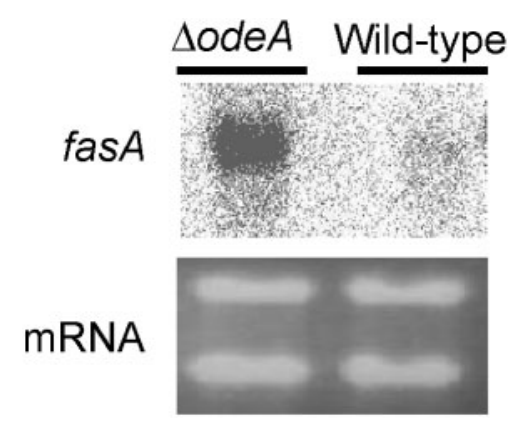

Fig. 2. Gene expression of $A$. parasiticus fas $A$, encoding the $\alpha$-subunit of fatty acid synthase, is upregulated in $\Delta$ ode $A$ compared to the wild-type.

\section{$\Delta^{12}$-desaturase mutation reduced rates of conidial spore germination and radial colony growth}

Germination of $\Delta$ ApodeA conidiospores was delayed, compared to the wild-type. Spores of the wild-type required $9 \mathrm{~h}$ growth in liquid GMM medium to achieve $100 \%$ germination rates, whereas $\Delta$ ApodeA required $14 \mathrm{~h}$. Although germination was delayed by $5 \mathrm{~h}$ in $\Delta$ ApodeA, we still observed $100 \%$ germination at this time, indicating that deletion of the odeA gene does not affect spore viability. $\Delta$ ApodeA growth was similarly reduced, compared to the wild-type. After 5 days, the mean colony diameter was $43 \cdot 88 \pm 0 \cdot 13 \mathrm{~mm}$ for wild-type $A$. parasiticus and $21 \cdot 98 \pm$ $0.55 \mathrm{~mm}$ for $\triangle$ ApodeA $(n=4)$, indicating a $50 \%$ reduction in $\Delta$ ApodeA growth, compared to the wild-type. These differences in colony diameter are significant $(P \leqslant 0 \cdot 05)$.

\section{$\Delta^{12}$-desaturase mutation reduced $A$. parasiticus conidial production and inhibited sclerotial development}

Conidial production of $A$. parasiticus $\Delta o d e A$ and wild-type strains after 3 days growth at $29^{\circ} \mathrm{C}$ in conditions of continuous light and 4 days growth in conditions of continuous darkness is shown in Fig. 3. The A. parasiticus $\Delta$ odeA

Table 2. Fatty acid composition of mycelia of $A$. parasiticus wild-type $(\mathrm{Wt})$ and $\Delta$ ode $A$

Analysis was carried out on mycelia grown for $72 \mathrm{~h}$ in liquid GMM under conditions of shaking and continuous light. Composition is given as ng FAME ( $\mu$ g lyophilized weight of mycelia) ${ }^{-1}$. 16:0, Palmitic acid; $16: 1$, palmitoleic acid; $18: 0$, stearic acid; $18: 1$, oleic acid; $18: 2$, linoleic acid; $18: 3$, linolenic acid. Values are means \pm SD of three replicates.

\begin{tabular}{|c|c|c|c|c|c|c|c|}
\hline \multirow[t]{2}{*}{ Sample } & \multirow[t]{2}{*}{ Total FAME } & \multicolumn{6}{|c|}{ Content of individual FAMEs (\%): } \\
\hline & & $16: 0$ & $16: 1$ & $18: 0$ & $18: 1$ & $18: 2$ & $18: 3$ \\
\hline $\mathrm{Wt}$ & $17 \cdot 1 \pm 0 \cdot 35$ & $18 \cdot 45 \pm 0 \cdot 22$ & $0 \cdot 32 \pm 0 \cdot 01$ & $13 \cdot 63 \pm 0 \cdot 37$ & $23 \cdot 5 \pm 0 \cdot 57$ & $40 \cdot 98 \pm 0 \cdot 88$ & $1 \cdot 1 \pm 0 \cdot 01$ \\
\hline$\Delta o d e A$ & $82 \cdot 1 \pm 0 \cdot 85$ & $7 \cdot 3 \pm 0 \cdot 1$ & $0 \cdot 5 \pm 0 \cdot 01$ & $2 \cdot 93 \pm 0 \cdot 01$ & $86 \cdot 23 \pm 0 \cdot 98$ & $0 \cdot 12 \pm 0 \cdot 01$ & $0 \cdot 16 \pm 0 \cdot 02$ \\
\hline
\end{tabular}




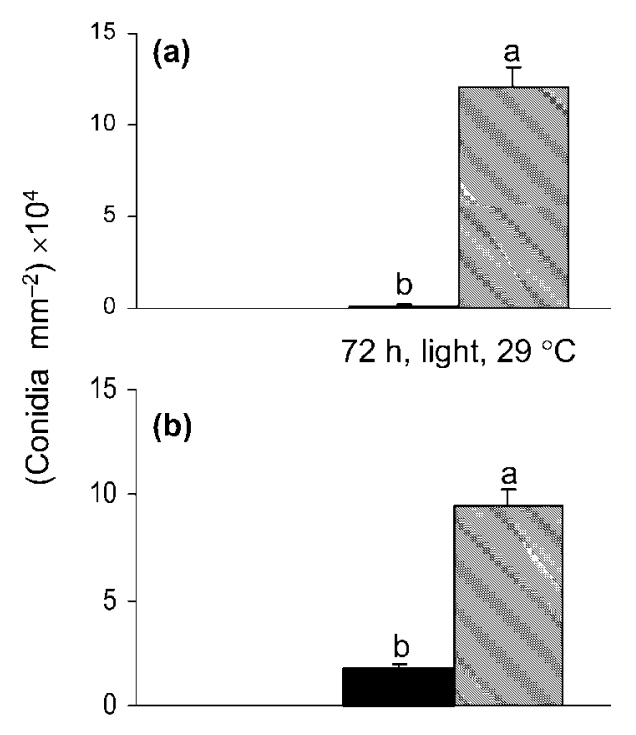

$96 \mathrm{~h}$, dark, $29^{\circ} \mathrm{C}$

Fig. 3. Conidial production of the $A$. parasiticus $\triangle$ ode $A$ mutant (filled column) and wild-type (hatched column). Values are means of four replicates. Columns with the same letter are not significantly different at a significance level of 0.05 .

mutant was significantly reduced in conidial development, compared to the wild-type strain, under both conditions.

Sclerotial development was also affected in the $\triangle$ odeA strain. On GMM agar plates, wild-type A. parasiticus produced a mean of $21 \cdot 5 \pm 1 \cdot 3$ sclerotia per plate $(n=4)$, whereas $\Delta o d e A$ produced no sclerotia. Sclerotia on GMM agar plates were counted after 7 days and these differences are significant $(P \leqslant 0 \cdot 05)$. On CAM plates, wild-type $A$. parasiticus produced a mean of $3 \cdot 5$ sclerotia per plate $(n=4)$, whereas $\triangle$ odeA produced no sclerotia. Sclerotia on CAM plates were counted after 14 days, but even after 1 month, $\triangle o d e A$ did not produce sclerotia (data not shown). Sclerotial mean values for CAM plates were taken from four out of a total of 15 plates per strain. On all CAM plates, the wild-type produced between two and six sclerotia, whereas $\Delta o d e A$ produced zero. Low sclerotial production by this $A$. parasiticus parental strain has been reported previously (Trail et al., 1995). By comparing results from the two growth media, it can be seen that the $\Delta o d e A$ mutation abolishes production of sclerotia and this effect is medium-independent.

\section{$\triangle$ odeA mutant strains were impaired in the colonization of peanut seed}

We assayed for the ability of $A$. parasiticus and A. nidulans $\triangle$ odeA strains, termed $\triangle$ ApodeA and $\triangle$ AnodeA, respectively, and the corresponding wild-type controls, to colonize two isogenic lines of peanut seed that differed only in oleic acid: linoleic acid ratios. Colonization was assessed in

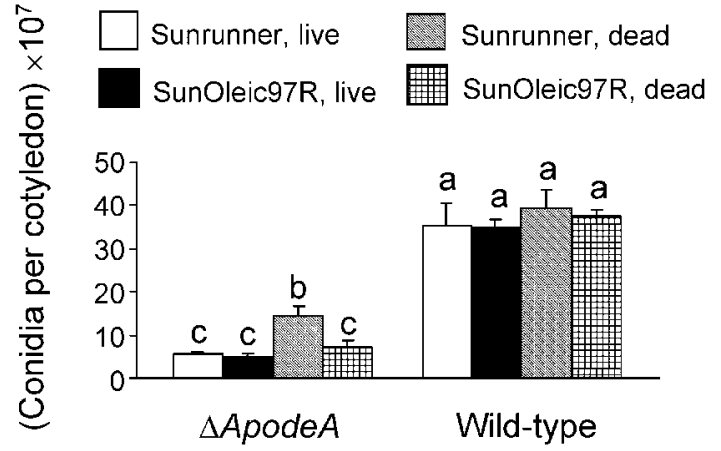

Fig. 4. Colonization of peanut seed by $A$. parasiticus $\Delta$ ode $A$ and wild-type strains. Strains were inoculated onto Sunrunner and SunOleic97R peanut-seed lines and incubated at $29^{\circ} \mathrm{C}$ in the dark for $96 \mathrm{~h}$. Dead and living seeds were used. Values are means of four replicates. Columns with the same letter are not significantly different at a significance level of 0.05 .

terms of conidial production on seed. Sunrunner has typical peanut seed oil content, containing $50 \%$ oleic acid and $30 \%$ linoleic acid [see Norden et al. (1985) and references therein]. SunOleic97R has increased oleic acid $(80 \%)$ and reduced linoleic acid (2-5\%) contents (Gorbet \& Knauft, 2000; Andersen \& Gorbet, 2002). These differences are due to recessive mutations of odeA orthologues in SunOleic97R (Knauft et al., 1993; Jung et al., 2000). Both live and dead seeds were used to assess the interactions between host seed and the fungus.

Wild-type A. parasiticus produced significantly more conidia $(P \leqslant 0 \cdot 05)$ on all peanut seeds than did $\triangle$ ApodeA. Wild-type $A$. parasiticus showed no significant differences $(P \leqslant 0 \cdot 05)$ in conidiation on any peanut seed, live or dead (Fig. 4), after $96 \mathrm{~h}$ growth. In control-plate growth tests, wild-type produced significantly more conidia than did $\triangle$ ApodeA (Fig. $3 b, P \leqslant 0 \cdot 05$ ) on GMM under dark conditions at $29^{\circ} \mathrm{C}$ for $96 \mathrm{~h}$.

Similar to A. parasiticus, wild-type A. nidulans produced more conidia on live seed than did the $\Delta$ AnodeA mutant. However, there were considerable differences in colonization between the two species. The A. nidulans strains produced markedly more conidia on dead seed than on live seed (Fig. 5a). On dead seed, $\triangle$ AnodeA and wild-type had the same rate of conidiation $(P \leqslant 0 \cdot 05)$. Both strains produced significantly more conidia $(P \leqslant 0 \cdot 05)$ on dead SunOleic97R than dead Sunrunner. On live seeds, conidial production of $A$. nidulans wild-type did not significantly differ on Sunrunner or SunOleic97R seeds. For $\Delta$ AnodeA, conidial production on live SunOleic97R and Sunrunner was reduced significantly $(P \leqslant 0 \cdot 05)$, compared to wildtype, with the greatest reduction seen on SunOleic97R. Interestingly, on control-growth test plates, $A$. nidulans $\triangle$ AnodeA produced significantly more conidia $(P \leqslant 0 \cdot 05)$ than wild-type on GMM under dark conditions at $29^{\circ} \mathrm{C}$ for 96 and 144 h (Fig. 5b). 
(a)

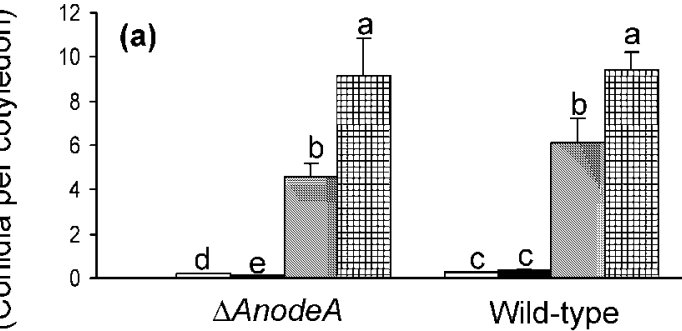
(b)

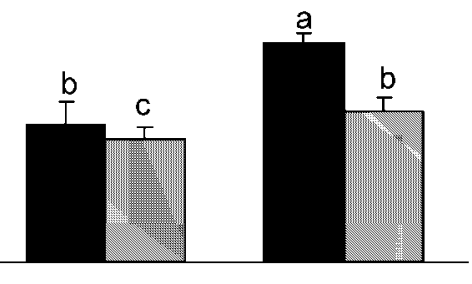

$96 \mathrm{~h}$

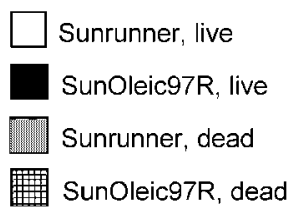

$144 \mathrm{~h}$
Fig. 5. Colonization of peanut seed by A. nidulans $\triangle \circ d e A$ and wild-type strains. (a) Cultures of $A$. nidulans $\triangle$ ode $A$ and wildtype strains were inoculated onto dead and live Sunrunner and SunOleic97R peanutseed lines. Strains were incubated for $96 \mathrm{~h}$ (for dead seed) or $144 \mathrm{~h}$ (for live seed) in the dark at $29^{\circ} \mathrm{C}$. Values are means of four replicates. (b) Cultures of $\triangle$ odeA (hatched column) and wild-type (filled column) strains were grown in the dark on GMM at $29^{\circ} \mathrm{C}$ for 96 and $144 \mathrm{~h}$ as a control for the colonization studies. Values are means of four replicates. Columns with the same letter are not significantly different at a significance level of 0.05.

\section{A. parasiticus $\triangle$ ode $A$ mutant was impaired in colonization of corn seed}

We also assayed for the ability of $A$. parasiticus wild-type and $\Delta o d e A$ strains to produce conidia on corn-seed lines. We chose Asgrow 404, a commercially available line that is susceptible to mycotoxin contamination in the field, and Tex6, a line with some resistance to mycotoxin production (Hamblin \& White, 2000). On dead seed, both strains had the same rates of conidiation and there was no significant difference $(P \leqslant 0 \cdot 05)$ between rates of conidiation on either corn-seed line (data not shown). However, on live seed, the A. parasiticus $\triangle$ odeA strain produced significantly fewer conidia $(P \leqslant 0 \cdot 05)$ than the wild-type on both seed lines (Fig. 6). Interestingly, both strains produced fewer conidia $(P \leqslant 0 \cdot 05)$ on Asgrow 404 seed lines than on Tex6 lines. Corn seed was not infected with A. nidulans strains.

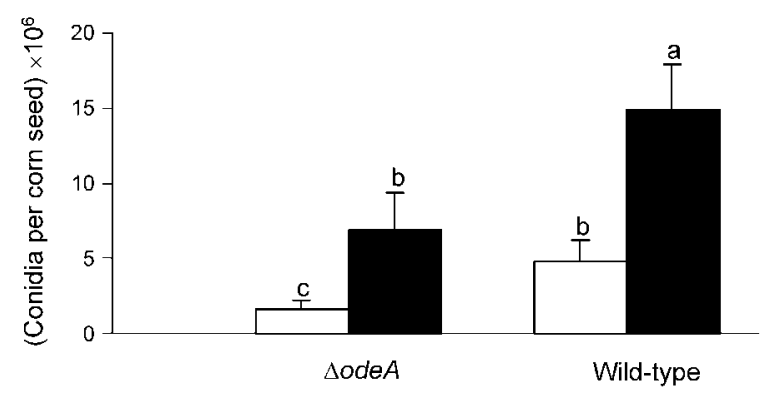

Fig. 6. Colonization of corn seed by $A$. parasiticus $\triangle$ ode $A$ and wild-type strains. Strains were inoculated onto Asgrow 404 (empty column) and Tex6 (filled column) lines and incubated for $96 \mathrm{~h}$ in the dark at $29^{\circ} \mathrm{C}$. Values are means of four replicates. Columns with the same letter are not significantly different at a significance level of 0.05 .

\section{DISCUSSION}

One potential area for the control of oilseed crop infection by mycotoxigenic Aspergillus species is to reduce production of the infectious particle, the conidium. Here, we studied the ability of Aspergillus desaturase mutants to colonize and produce conidia on seed, partly to investigate the suitability of focusing on fatty acid metabolism as a means to reduce spore production.

The odeA gene, which encodes a $\Delta^{12}$-oleic acid desaturase that is involved in converting oleic acid to linoleic acid, was identified and disrupted in A. parasiticus. Mycelia of the resulting $\Delta$ odeA mutant $(\triangle \mathrm{ApodeA})$ had a similar fatty acid content to the $A$. nidulans $\triangle$ odeA mutant $(\triangle$ AnodeA; Calvo et al., 2001), with dramatically increased oleic acid content and reduced contents of linoleic and linolenic acids (Table 2). In addition, total fatty acid content accrued in $\triangle$ ApodeA to a level five times greater than that seen for wild-type. Calvo et al. (2001) reported a similar increase in the total fatty acid content of $\Delta$ AnodeA and attributed it to a lack of polyunsaturated fatty acid inhibition of fas $\mathrm{A}$ gene expression, leading to upregulation of fatty acid biosynthesis. The increase in fatty acid content in $\triangle$ ApodeA (Table 2), coupled with increased fasA gene expression (Fig. 2), suggests that the feedback inhibition mechanism is conserved in A. parasiticus.

Linoleic acid is a major constituent of fungal lipid and commonly comprises $30-50 \%$ of the total fatty acid content in mycelia and conidia of aspergilli (Table 2; Singh \& Sood, 1973; Sood \& Singh, 1973; Rambo \& Bean, 1974; Budinska et al., 1981; Evans et al., 1986; Chattopadhyay et al., 1987; Calvo et al., 2001). Several studies have shown that various unsaturated fatty acids affect Aspergillus developmental processes (Mazur et al., 1990, 1991; Calvo et al., 1999), whilst ratios of unsaturated: saturated fatty 
acids are important for Aspergillus and Mucor development (Calvo et al., 1999; Khunyoshyeng et al., 2002). In A. nidulans, specific individual fatty acids are also required at different stages of development. For example, Evans et al. (1986) reported that mycelial linolenic acid concentration was prominent in 1-day-old cultures, but declined to trace levels in cultures older than 3 days. The $\Delta$ ApodeA mutant was altered in all these parameters of fatty acid metabolism, displaying reduced production of linoleic and linolenic acids and altered ratios of unsaturated:saturated fatty acids. It was consequently defective in rates of spore germination, colony growth and development. In a parallel study, a $\Delta o d e A$ mutation that was generated in an $O-$ methylsterigmatocystin-accumulating strain of A. parasiticus, SRRC 2043, also resulted in altered conidiation and sclerotial development (Chang et al., 2004). This supports our conclusion that the reduction in conidiation and abolishment of sclerotial production seen for $\triangle$ ApodeA compared to the wild-type is due solely to aberrant polyunsaturated fatty acid metabolism and serves to emphasize the genetic connection between fatty acid metabolism and fungal development.

The role of fatty acid metabolism in the plant-fungal interaction was studied by using two peanut lines and two corn lines. Because of an increasing interest in developing peanut seed with higher oleic and lower linoleic acid content, due to the value of monounsaturated fatty acids in the diet (Massaro et al., 1999), and considering the importance of fatty acids in Aspergillus development, we investigated whether Aspergillus species differentially colonized two near-isogenic commercial peanut lines with altered fatty acid content. One line is SunOleic97R, which contains high amounts of oleic acid $(80 \%)$, but reduced amounts $(2-5 \%)$ of linoleic acid and undetectable amounts of linolenic acid. Another is Sunrunner, which contains $50 \%$ oleic acid and $30 \%$ linoleic acid (Holbrook et al., 2000). For the corn lines, we chose Asgrow 404 and Tex6, lines that exhibit some susceptibility and resistance to aflatoxin contamination, respectively, in the field (Hamblin \& White, 2000). Conidiation of wild-type A. parasiticus and $A$. nidulans on live peanut seed did not differ between these two lines, indicating that the reduced amount of linoleic acid in SunOleic97R is still sufficient to induce and support conidiation. In contrast, the $\Delta o d e A$ mutants of A. parasiticus and A. nidulans exhibited a significant reduction in conidiation on live peanut seed (Figs 4 and 5), indicating a direct or indirect role for the odeA gene in fungal development on peanut seed. On corn seed, the A. parasiticus $\Delta o d e A$ strain also produced fewer conidia on both corn lines, compared to the wild-type (Fig. 6). Wild-type A. parasiticus did not produce significantly different amounts of conidia on dead Asgrow 404, live Tex6 and dead Tex6 seed (data not shown). However, conidial production of wild-type A. parasiticus was reduced significantly on live Asgrow $404(P \leqslant 0 \cdot 05)$, suggesting an inhibitory effect of this line that is not seen for Tex6. This observation is interesting as it contrasts with the situation observed in the field, where the Asgrow 404 line is more susceptible to aflatoxin contamination, whereas Tex6 has some resistance (Hamblin \& White, 2000). Nonetheless, reducing fungal colonization and sporulation would be a prerequisite for curbing aflatoxin contamination of crops. Taken together, these results suggest that whilst altering the fatty acid content of oil seeds might not reduce Aspergillus conidiation and probable spread of the pathogen, inactivation of the fungal odeA gene may do so. Therefore, a promising strategy for controlling contamination could be the selective targeting and inactivation of fungal $\Delta^{12}$-desaturases by fungicides.

Wild-type A. parasiticus did not produce different amounts of conidia on either live or dead peanut lines, suggesting that the plant defensive response of live seed is not effective to reduce the development of this fungus. In contrast, all A. nidulans strains produced more conidia on dead seed than on live seed, even when growth on live seed was prolonged. This indicates the effectiveness of the plant defensive response against $A$. nidulans, which is not a pathogen of peanut in nature.

This study shows that pathogenic differences exist between A. nidulans and A. parasiticus and that loss-of-function desaturase mutants of both species are impaired in colonization abilities. One future course of our study will be an in-depth analysis of the interactions that are involved in the colonization of oilseed crops by $A$. nidulans and $A$. parasiticus and the closely related A. flavus. By using available genomic and expressed sequence tag resources of A. nidulans and A. flavus, coupled with microarray technology, subtractive libraries and proteomics, one could further dissect the Aspergillus-seed interaction to identify the molecular bases for differences in colonization by saprophytic, pathogenic and mutant aspergilli as new targets for reducing the infestation of oilseed crops by these fungi.

\section{ACKNOWLEDGEMENTS}

We thank Dr Brian Fox and Mr Enrique Gomez for assistance with fatty acid analysis, Dr John Linz for A. parasticus vectors and cosmid libraries and Dr Corley Holbrook for providing Sunrunner and SunOleic97R seeds. This work was funded by NRI 2001-35319-10996 and USAID under the Peanut CRSP grant LAG-G-00-96-90013-00 to N.P. K and supported in part by a specific cooperative agreement, 58-6435-081, with USDA-ARS.

\section{REFERENCES}

Andersen, P. C. \& Gorbet, D. W. (2002). Influence of year and planting date on fatty acid chemistry of high oleic acid and normal peanut genotypes. J Agric Food Chem 50, 1298-1305.

Bewley, J. D. \& Black, M. (1985). Seeds: Physiology of Development and Germination, p. 16. New York: Plenum.

Bligh, E. G. \& Dyer, W. J. (1959). A rapid method of total lipid extraction and purification. Can J Med Sci 37, 911-917. 
Budinska, O., Kubin, V., Franek, J., Julak, J. \& Panos, J. (1981). A lipopolysaccharide from Aspergillus flavus conidia. Folia Microbiol (Praha) 26, 212-216.

Burow, G. B., Nesbitt, T. C., Dunlap, J. \& Keller, N. P. (1997). Seed lipoxygenase products modulate Aspergillus mycotoxin biosynthesis. Mol Plant Microbe Interact 10, 380-387.

Burow, G. B., Gardner, H. W. \& Keller, N. P. (2000). A peanut seed lipoxygenase responsive to Aspergillus colonization. Plant Mol Biol 42, 689-701.

Calvo, A. M., Hinze, L. L., Gardner, H. W. \& Keller, N. P. (1999). Sporogenic effect of polyunsaturated fatty acids on development of Aspergillus spp. Appl Environ Microbiol 65, 3668-3673.

Calvo, A. M., Gardner, H. W. \& Keller, N. P. (2001). Genetic connection between fatty acid metabolism and sporulation in Aspergillus nidulans. J Biol Chem 276, 25766-25774.

Chang, P.-K., Wilson, R. A., Keller, N. P. \& Cleveland, T. E. (2004). Deletion of the $\Delta^{12}$-oleic acid desaturase gene of a non-aflatoxigenic Aspergillus parasiticus field isolate affects conidiation and sclerotial development. J Appl Microbiol (in press).

Chattopadhyay, P., Banerjee, S. K., Sen, K. \& Chakrabarti, P. (1987). Lipid profiles of conidia of Aspergillus niger and a fatty acid auxotroph. Can J Microbiol 33, 1116-1120.

Evans, J. L., Moclock, M. A. \& Gealt, M. A. (1986). The fatty acid composition of the conidia and mycelia of the fungus Aspergillus nidulans. Can J Microbiol 32, 179-181.

Geiser, D. M., Timberlake, W. E. \& Arnold, M. L. (1996). Loss of meiosis in Aspergillus. Mol Biol Evol 13, 809-817.

Gorbet, D. W. \& Knauft, D. A. (2000). Registration of 'SunOleic 97R' peanut. Crop Sci 40, 1190-1191.

Hamblin, A. M. \& White, D. G. (2000). Inheritance of resistance to Aspergillus ear rot and aflatoxin production of corn from Tex6. Phytopathology 90, 292-296.

Holbrook, C. C., Wilson, D. M., Matheron, M. E., Hunter, J. E., Knauft, D. A. \& Gorbet, D. W. (2000). Aspergillus colonization and aflatoxin contamination in peanut genotypes with reduced linoleic acid composition. Plant Dis 84, 148-150.

Jung, S., Powell, G., Moore, K. \& Abbott, A. (2000). The high oleate trait in the cultivated peanut [Arachis hypogaea L]. II. Molecular basis and genetics of the trait. Mol Gen Genet 263, 806-811.

Khunyoshyeng, S., Cheevadhanarak, S., Rachdawong, S. \& Tanticharoen, M. (2002). Differential expression of desaturases and changes in fatty acid composition during sporangiospore germination and development in Mucor rouxii. Fungal Genet Biol 37, 13-21.
Knauft, D. A., Moore, K. M. \& Gorbet, D. W. (1993). Further studies on the inheritance of fatty acid composition in peanut. Peanut Sci 20, 74-76.

Massaro, M., Carluccio, M. A. \& De Caterina, R. (1999). Direct vascular antiatherogenic effects of oleic acid: a clue to the cardioprotective effects of the Mediterranean diet. Cardiologia 44, 507-513.

Mazur, P., Meyers, H. V., Nakanishi, K., El-Zayat, A. A. E. \& Champe, S. P. (1990). Structural elucidation of sporogenic fatty acid metabolites from Aspergillus nidulans. Tetrahedron Lett 31, 3837-3840.

Mazur, P., Nakanishi, K., El-Zayat, A. A. E. \& Champe, S. P. (1991). Structure and synthesis of sporogenic psi factors from Aspergillus nidulans. J Chem Soc Chem Commun 20, 1486-1487.

Norden, A. J., Gorbet, D. W. \& Knauft, D. A. (1985). Registration of 'Sunrunner' peanut. Crop Sci 25, 1126.

Payne, G. A. (1992). Aflatoxin in maize. Crit Rev Plant Sci 10, 423-440.

Rambo, G. W. \& Bean, G. A. (1974). Sterols and fatty acids of aflatoxin and non-aflatoxin producing isolates of Aspergillus. Phytochemistry 13, 195-198.

Sambrook, J., Fritsch, E. F. \& Maniatis, T. (1989). Molecular Cloning: a Laboratory Manual, 2nd edn. Cold Spring Harbor, NY: Cold Spring Harbor Laboratory.

Singh, J. \& Sood, M. G. (1973). Component fatty acids of Aspergillus terreus fat. J Am Oil Chem Soc 50, 485-486.

Skory, C. D., Chang, P.-K., Cary, J. \& Linz, J. E. (1992). Isolation and characterization of a gene from Aspergillus parasiticus associated with the conversion of versicolorin A to sterigmatocystin in aflatoxin biosynthesis. Appl Environ Microbiol 58, 3527-3537.

Sood, M. G. \& Singh, J. (1973). The component fatty acids of Aspergillus ochraceus fat. J Sci Food Agric 24, 1171-1174.

Trail, F., Mahanti, N., Rarick, M., Mehigh, R., Liang, S.-H., Zhou, R. \& Linz, J. E. (1995). Physical and transcriptional map of an aflatoxin gene cluster in Aspergillus parasiticus and functional disruption of a gene involved early in the aflatoxin pathway. Appl Environ Microbiol 61, 2665-2673.

Tsitsigiannis, D. I., Zarnowski, R. \& Keller, N. P. (2004). The lipid body protein, PpoA, coordinates sexual and asexual sporulation in Aspergillus nidulans. J Biol Chem 279, 11344-11353.

Wilson, R. A., Gardner, H. W. \& Keller, N. P. (2001). Cultivardependent expression of a maize lipoxygenase responsive to seed infesting fungi. Mol Plant Microbe Interact 14, 980-987.

Wilson, R. A., Chang, P.-K., Dobrzyn, A., Ntambi, J. M., Zarnowski, R. $\&$ Keller, N. P. (2004). Two $\Delta 9$-stearic acid desaturases are required for Aspergillus nidulans growth and development. Fungal Genet Biol 41, 501-509. 